\title{
Automatic Analysis of Movies for Content Characterization
}

\author{
Sher Muhammad Doudpota ${ }^{1}$, Sumanta Guha ${ }^{2}$
}

\begin{abstract}
This paper describes an approach to automatically detect music and song sequences in movies. Particularly, the system is designed to identify songs in movies, as well as their genre, playback singer and on-screen performing actors. We define the so-called song grammar that a musical sequence typically follows by means of a finite state machine.
\end{abstract}

Keywords-Action, content characterization, dialogue, finite state machine, movie, song.

\section{INTRODUCTION}

A Substantial portion of the world's population enjoys movies as a great source of entertainment. This results in an extensive market for movies throughout the world. Bollywood, Hollywood and Lollywood are few names to mention in a long list of film producing industries, which produce hundreds of movies for their millions of audience every year.

Regardless of which industry produces a movie, it contains a mix of scenes belonging to different categories, such as love scenes, action scenes, conversation scenes, music and songs. All these types of scenes contribute in moving the story forward. Every scene in a movie has certain audio-visual features that differentiate it from other scenes. For example, in a conversation scene, usually there is little actors' movement, low or no background music and long shot duration with repetitive shots. On other hand, an action scene might have rapid movements, lot of sound effects and short shot duration with less or no repetition of shots. Although never codified, there are well-accepted movie making rules for every type of scene which are followed worldwide and, if broken, will confuse the audience.

In a Bollywood movie, typically, along with above mentioned scene types, there is also a lot of music and songs. Almost every Bollywood movie contains 4 to 10 songs and every song lasts 3 to 10 minutes. Music in Indian and south Asian cinema is a substantial revenue generator, with the music rights alone accounting for 4-5\% of the net revenues generated by a film in India. Commercially, film music accounts for $48 \%$ of India's net music sales [7].

Songs in movies are mixed depending on their genre. For example, a sad scene might be scored by a song belonging to sad genre, whereas, a love scene might be accompanied by a romantic song. Generally, a movie song falls in one of four categories: romantic or slow song, sad or tragic song, pop or fast-paced song, and general purpose song mainly played during some celebration-like event in movies. Like movie

School of Engineering and Technology, Asian Institute of Technology, Bangkok, Thailand ${ }^{1,2}$ email: $\left\{\right.$ sher.muhammad.doudpota ${ }^{1}$ guha $\left.^{2}\right\} @$ ait.ac.th making, song making does also have certain well defined rules which are followed while making any type of song, belonging to above mentioned genres.

Songs in Bollywood movies are mostly filmed on the main actors of the movie. However, it is not sung by that actor, rather there are playback singers who sing the songs for movies. There are a few popular playback singers in Indian movies, namely, Udat Narayan, Kumar Sanu, Lucky Ali, Lata Mangeshkar, Anuradha Paudwal, Kavita Kirshnamurthy etc. It is quite interesting to note that these names have a big role to play in the success of a movie. In India, a movie is considered a hit if its music leaves an impact on listeners. This is the reason why, not only on-screen actors are considered stars in Indian movies, but playback singers also have stardom.

A song in a movie is always accompanied by background music. Therefore, in order to locate a song in a video, we should search parts where there is music. There are various recent attempts in the literature to extract musical parts from a video.

HARB [4] breaks a video in four classes, namely speech, music, silence and noise. A Silence Crossing Rate in [4] is defined as the number of times the energy falls below the silence level. Experiments show that SCR is 5 to 10 for speech in one second window. However it is different for music and noise. Figure 1 shows this difference in speech and music energy envelops. It shows that there are many instances in speech when signal level crosses the silence threshold, where as in music this is hardly seen.

Scheirer in [5] gives a comprehensive list of features that can be efficiently used in the discrimination of music and speech. This list includes percentage of low energy frames, spectral roll off point, spectral centroid, and zero crossing rate.

Lu [6] discusses a K-nearest neighbor classification algorithm for classifying audio according to its type or speaker identity. In this approach, audio is segmented and classified into speech, music, environment sound and silence. A two step classification is presented. In the first step, audio is broken in two classes, speech and non-speech. In the second step, the non-speech class is further classified into music, environment sound and silence using a rule based classification.

There are many audio features that can be used for classifying audio in music and non-music classes. However, out of all these, the audio feature that is mostly used in [8][10][11] for music and non-music discrimination is zero crossing rate.

Various approaches found in recent litrature for indexing movies assume only two types of scenes in a movie, mainly, dialogue and action scenes.

This assumption might hold true in Hollywood movies, 


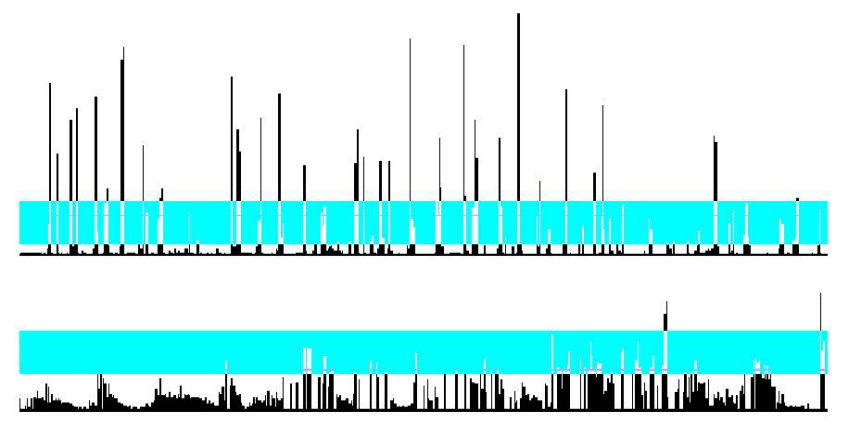

Fig. 1. Energy Envelope Shape for Speech (top) and Music (bottom)

but in Bollywood and Lollywood movies, music and songs are important integral part of a movie. So, while indexing a movie, music and songs should also be considered as a desired retrievable unit of a movie.

Apparently, a song from a movie can be extracted easily by locating music parts in a movie, because songs in a movie are always accompanied by background music. As presented above, there are many proposals [4][5][6][7][11][12] that can be used to locate music segments in a movie. However, when studied closely, in a Bollywood movie, it is not only song which is accompanied in music parts of movie, but there are also dialogue sequences and action sequences which come with background music. This shows that in order to extract a song from a movie there should be some mechanism to differentiate between music with singing voice and music with speech.

In this paper, we propose a comprehensive system that exploits Finite State Machine to extracts music and songs from movies as well as three additional components to identify genre, on-screen performing actor and playback singer of extracted songs.

The rest of the paper is organized as follows. Section II gives an overview of our proposed system. Section III discusses how music is seperated from non-music parts of a movie. The extraction of song from music part is explained in Section IV. The remaining components of the system, song genre identifier, playback singer identifier, and on-screen actor identifier are discussed in section V, VI, and VII respectively. Finally, Section VIII concludes the paper.

\section{System OVERVIEW}

An abstract model of proposed system is depicted in Fig 2. It accepts a movie or an array of movies as input of Music-NonMusic Separator component of the system. This component separates parts of movie where there is presence of music from parts where there is no music.

As discussed in the introduction, the presence of music is evidence that there might be songs in these parts. So the part of movie where there is no music is fed as input to the dialogue/action scene detector. The remaining part of movie where there is presence of music has three possible scenes, namely, dialogue scenes, action scenes and songs. The rest of the components of system have to work on this part of movie.

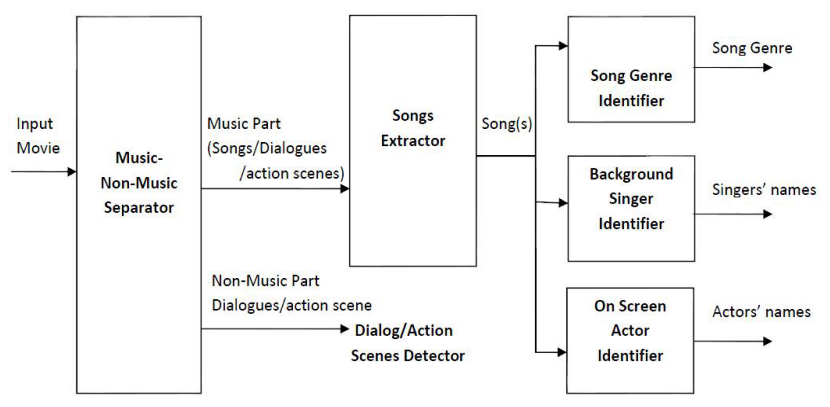

Fig. 2. Abstract Model of Proposed System

The second component of the proposed system is Song Extractor that accepts music part of movie and produces $n$ number of audio/video files where $n$ represents number of songs in input movie. It rejects the scenes where there are either dialogue or action sequences and outputs only songs. The extracted songs from Song Extractor are fed in remaining three components.

The genre of a song is identified by Song Genre Identifier. It uses different characteristics of songs in order to accomplish genre identification. Genre of a song could be sad or tragedy, love or romantic, pop music, and general.

The playback singers of extracted songs are identified by next component i.e. Playback Singer Identifier. It accepts songs at input and produces the names of singers as output.

The last component of the system is On-screen Actor Identifier. This component accepts song as input and produces the names of the actor performing the song.

\section{Music Non-Music SeParator}

This component of the system is responsible for discriminating music and non-music scenes in input movie. As discussed earlier, a song must be accompanied by background music. So there is no reason to search for songs in scenes where there is no music.

Recent research has shown that there are definite audio features that help to distinguish between music and speech or non-music frames in an audio/video file. Out of a big list of audio features, Zero Crossing Rate, Root Mean Square (RMS), Short Time Energy Ratio, and Spectral Flux has proved to produce best results. Scherer [5] performed various experiments on these features for different classification models including $G M M, B P-A N N$ and $K N N$. When using a window of same size $(2.4 \mathrm{sec})$ for different classification models, the reported performance is up to $99.6 \%$ [6].

In our work, in order to seperate music-accompanied parts from non-music, we use Scherer's [5] approach of classification based on GMM with window size of 2.4. It gives us very promising results, as good as $99.6 \%$.

\section{SONG EXTRACTOR}

This component of the system receives the music part as input from Music Non-Music Separator. As discussed earlier, in this part of the movie, there could be many occurrences of 

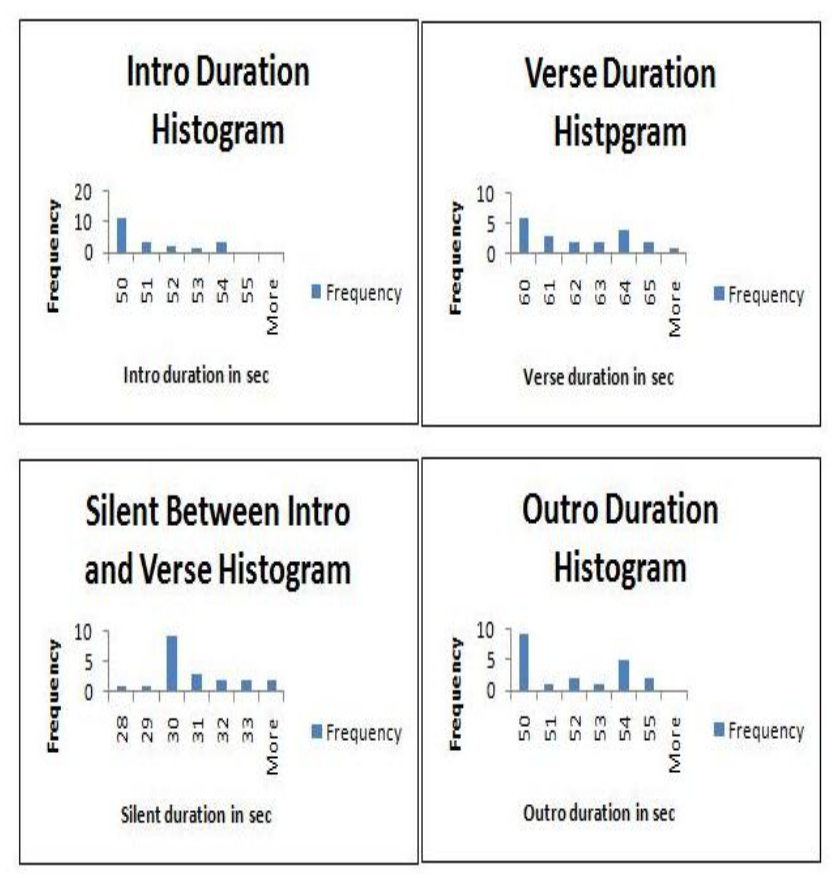

Fig. 3. Frequency vs Duration Histogram for intro, verse, silent between intro and verse, and outro

dialogue scenes, action scenes and songs. Out of these three types of scenes, the Songs Extractor is responsible to extract songs and discard other two types of scenes.

Regardless of genre of song, every song follows certain rules of composition as well as lyrics writing. This component of system exploits these rules to locate song in music part of movie. A song is always composite of different elements including intro, verse, pre-chorus, chorus, bridge and outro.

It has been observed through study of many songs that a Bollywood or Lollywood song always starts with an intro, followed by any optional element from pre-chorus, chorus, bridge, and contains 1 to 4 occurrences of verse. Pre-chorus, chorus, and bridge are normally pure music components, whereas, intro, outro and verse are human singing voice. The singing voice in a song appears regularly following a well defined pattern.

Suppose we represent chorus, pre-chorus, bridge as $S$ i.e. silent segment where there is no singing voice (although music is there), a song can be represented by following regular expression,

$$
\mathbf{I}^{+}\left(\mathbf{S}(\mathbf{V S})^{+} \mathbf{I}\right)^{+} \mathbf{O}
$$

Where:

$I \rightarrow$ Inro,

$S \rightarrow$ Silent (Chorus, Pre-chorus, Bridge),

$V \rightarrow$ Verse,

$O \rightarrow$ Ouro.

The regular expression 1 shows that a song has an inro that is sung at least once and repeated for some times which in most cases is 2 to 3 times, followed by a silent part combined with verse and another silent part. This silent, verse and silent pattern in a song appears at least once and may present for

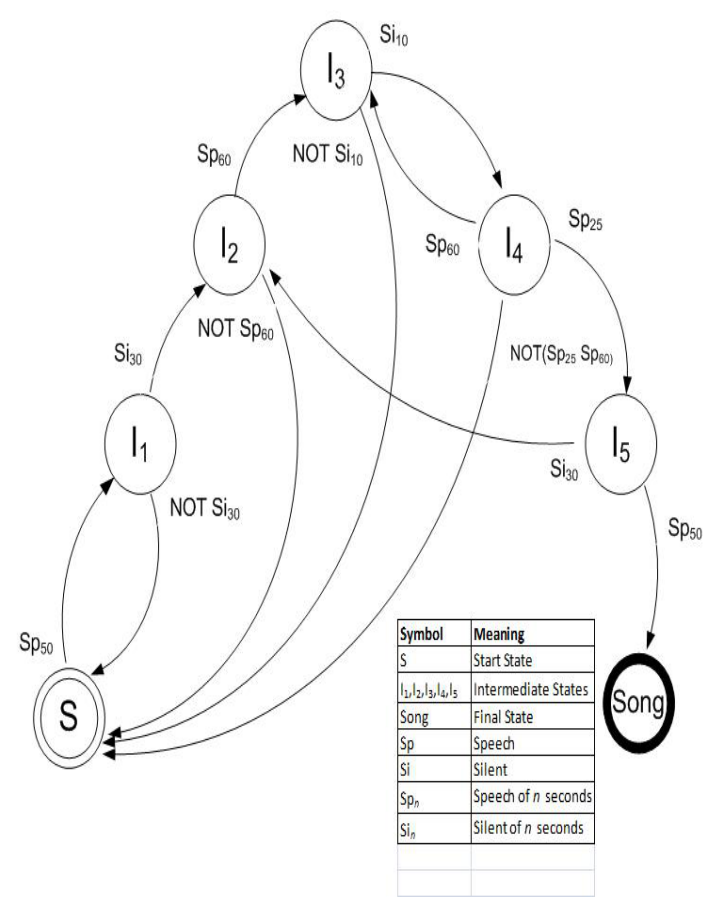

Fig. 4. Finite State Machine for Song Extraction

a total of 1 to 4 times depending on length of song. The last part of a song is outro that is used to exit from a song. Outro is also played at least one time followed by its repetition 1 or more times.

Through the analysis of various songs, it has been observed that intro of a song is always around 50 seconds, whereas silent before verse is 30 seconds and every verse has a duration of 60 seconds. Finally, length of outro is generally same as intro. For the analysis purpose, we selected 20 most popular songs of Bollywood movies and observed duration of intro, verse, silent between intro and verse, and outro. Fig 3 shows frequency histograms of these four song components. The difference between intro played at start and one that is played after a verse is that, the latter has as much as half duration than earlier.

\section{A. Finite State Machine Operation}

In order to detect a song from music part of movie, we have to search for speech patterns based on regular expression given in expression 1. Fig 4 shows a Finite State Machine that can be used to detect sequences of audio-visual frames that might be involved in a song scene. The operation of proposed FSM is outlined as follows,

- The FSM remains in start state until a consecutive 50 seconds speech with background music is observed. In this case, machine moves to first intermediate state i.e. $I_{1}$. Note that these 50 seconds might represent intro of a song or a normal speech in an action or dialogue scene.

- While in state $I_{1}$, machine reads next 30 seconds, if during next 30 seconds, only music is observed, machine moves to next intermediate state i.e. $I_{2}$, otherwise machine reverts and comes back to start state. At this 
stage, machine assumes it has encountered intro of a song followed by silent between intro and verse. So now it looks for first verse of song.

- A verse starts with 60 seconds of speech. If found, machine moves to next intermediate state $I_{3}$, otherwise it reverts back to start state assuming that there is either dialogue or action scene.

- When machine is in state $I_{3}$, it means that it has found intro, silent after intro, and verse. A verse in song is generally repeated twice or thrice with a silent interval of $10 \mathrm{sec}$ between each repetition. So the next possibility in a song is a silent segment of 10 seconds. If found, machine moves to state $I_{4}$, otherwise reverts back to start state assuming wrong detection of song.

- After 10 seconds of silent, if a verse is repeated, there should be another 60 seconds speech in state $I_{4}$ that causes move back to state $I_{3}$. But if verse is already repeated for required number of time then it normally would be followed by half intro part that is a speech segment of 25 seconds. If observed it moves to state $I_{5}$ where it is assumed that a verse has been completed.

- When machine is in state $I_{5}$, it means at least a verse along with intro has been played. There are two possibilities at this stage,

1) There are more verses left in song. In this case, there should be a silent of 30 seconds followed by next verse. So if a silent of 30 seconds found, machine reverts back to state $I_{2}$ to search for a pattern of next verse

2) Otherwise, enough verses have been found so there should be a complete final repetition of intro at the end, i.e. an outro. So there should be another speech of 50 seconds. If observed, machine moves to final state that concludes correct detection of song.

\section{SONG GENRE IDENTIFIER}

This component of system accepts a song at input and decides about the genre of input song. The genre of a song could be pop or fast music song, romantic song, sad song and general song.

According the rules of song making, a pop song generally has dance of involved actors, so naturally there would more movement in these type of songs than any other type. In order to excite pop-music songs' veiwers, the shot duration is also kept very short.

On other hand, for sad songs, there is no any dance, resulting in low actors' movement. In sad songs, camera mostly focuses on sad expressions of actor, that makes a long shot duration.

Finally, romantic and general songs are very close in their features so combined in one category. These type of songs generally has meduim shot duration and meduim to high movement of actor. Table 1 summarizes the rules for different songs' genre.

\section{Playback Singer IDENTIFIER}

This component of the proposed system accepts a song at input and identifies singer of the song. In film industry, there is
TABLE I

SONG GENRE IDENTIFICATION RULES

\begin{tabular}{ccc}
\hline \hline Actors' Movement & Shot Duration & Song Genre \\
\hline High & Low & Fast or Pop Song \\
Meduim & Meduim & Romantic or Genreal Song \\
Low & Long & Sad \\
\hline
\end{tabular}

a limited number of playback singers. It would be reasonable to expect singer of any new song from that pool of limited but well known singers in industry.

Keeping the fact in view, this component is broken in two phases, in phase 1, profile of each possible singer is constructed and stored in database. This phase is known as training phase, in which record of all singers is stored in database. In 2nd phase, audio features of input song are extracted and machted with singers available in database. The most likely matched singer profile would be assigned as singer of input song. Two phases are briefly discuessed in following sections.

\section{A. Singers' Profile Construction Phase}

A profile of a singer represents his/her voice in our system. The profile construction is like training phase of system in which we keep record of all the singers in film industry. Following steps are performed for singer's profile contruction,

- $N$ number of songs are manually selected for a singer where $N$ represents different genres in which singer has sung songs.

- Vocal and non-vocal parts of song are seprated manually. Because singing voice would be present in vocal parts, so non-vocal part, that consists only music, is discarded.

- Audio features are extracted from vocal parts (singing voice with music) and stored in database as profile of singer.

The first two steps are performed manually, however, 3rd step i.e. features extraction need some explantaion. Audio features of each selected vocal part of song are extracted and represent voice of a singer. There are many audio features which can be used to uniquely represent voice of singer. MFCC (MelFrequency Cepstral Coefficients) and $L P C$ (Linear Predictive Coding Coefficients) are two audio features mostly used in research for speeker identification and speech recoginition. Previous studies [8] [9] [10] [11] [12] has shown that performance of these two features in singer identification is as good as $82 \%$ accuracy. In our work, we are using these two audio features for uniquily representing singer's voice in singers' database.

\section{B. Singer Identification Phase}

In this phase of singer identification, actual identification of singer takes place based on training data set up in previous phase. In order to accomplish this task, following steps are performed on input song,

1) Sample of singing voice is extracted form start of input song. 
2) Audio features (MFCC and $L P S$ ) are extracted from sample of input song and compared with all the profiles of singers available in training database.

3) The best match in step 2 is assigned as singer of input song.

Note that in training phase, singing voice can be seperated from only-music part manually, but in 2nd phase it has to be done automatically.

It has been noticed in Bollywood and Lollywood songs that they normally start with only-music. The only-music part sustains for few seconds normally in the range of 5 to 20 , followed by singing voice of singer. In our study, we need the sample of singing voice, so we have to skip the music only part of input song. This can be done by observing certain audio features like signal enrgy, zero crossing rate, and spectral flux of input songs.

It is obvious that when there is only music in input song, its signal energy level would remain stable, but as singing voice is introduced in song, there would abtrupt enhancement in signal's energy. This enhancement in signal energy could be identified as introduction of singing voice in input song. In similar way, the variations in spectral flux and zero crossing would be stable when there is only music. When singing voice is introduced, these variations would be higher. The change in variations of spectral flux and zero crossing could also be used as evedance of singing voice induction.

Once there are sufficient evidances of singing voice availability, we take a sample of input audio for 25 seconds, that would be used in next steps for comparision with already existing audio features of singers' voice.

\section{ON-SCREEN ACTOR IDENTIFIER}

This component of system is responsible for identifying onscreen actor performing on song. It accepts a song at input and produces name of actor(s) performing on that input song. There is a limited number of actors who are popular at a time in a film industry. This component of the system keeps face record of those popular actors in database and follows following steps in order to identify actors performing on input song,

- Start of song is detected and all the faces in first 25 seconds' frames are captured.

- The faces extracted from first 25 seconds' frames of a song are compared with already existing faces in database.

- The best match faces are recognized as actors performing on that input song.

The reason to capture faces only from first 25 seonds is that in Bollywood and Lollywood movies, at the start of a song, most probably face of main actor appears domanantly on screen. Most of the times, only main actors' face is visible on screen. However, there might be few other faces aroud the actor, but our system would discard them because there would not be record of those unpopular faces in system's database.

\section{CONCLUSION}

Music and Songs are integral part of movies produced at Bollywood and Lollywood. The success of a movie's music almost guaranties the success of film itself. For a viewer, who is interested in only music of a movie, it is difficult to locate a song of his choice from a movie of 2 to 3 hours. The only currently available option for him is to locate it manually using forward and reverse mechanisims. The genre, playback singer, and on-screen actor performing on a song are the basic criterion for a viewer to select a song from a movie. This paper proposes a system that exploits audio-visual low level features to extract songs from a movie based on above criterion.

\section{REFERENCES}

[1] A. W. M Smeulders, S. Santini, Content Based Image Retrieval at the End of Early Years, IEEE Transactions on Patterns Analysis and Machine Intelligence, Vol. 22 No. 12, pp. 17-49, December 2000.

[2] A. K. Jain, A. Vailaya, X. Wei, Query by Video Clip, Multimedia System, Vol 7, No. 5, pp 369-384, September 1999

[3] K. Shirhama, K. Uehara, Query by Shots: Retrieving Meaningful Events Using Multiple Queries and Rough Set Theory, In Proceedings of Ninth ACM International Conference on Multimedia Data Mining and Knowledge Discovery from Databases, Las Vegas, August 2008.

[4] H. Harb, L. Chen, J. Auloge, Speech/Music/Silence and Gender Detection Algorithm, In Proceedings of the 7th International Conference on Distributed Multimedia Systems (DMS 01), May 2001.

[5] E. Scheirer, M. Slaney, Construction and Evaluation of Robust Multifeature Speech/Music Discriminator, Acoustics, Speech, and Signal Processing (1997), IEEE International Conference on Munich, Germany (Apr. 21-24, 1997), pp. 1331-1334

[6] L. Lu, H. J. Zhang, H. Jiang, Content Analysis for Audio Classification and Segmentation, IEEE Transaction on Speech and Audio Processing, pp 504-516, October 2002.

[7] http://en.wikipedia.org/wiki/Bollywood

[8] . Sheen, J. Shephered, B. Cui, A Novel Framework for Efficient Automatic Singer Identification in Larger Music Databases, ACM Transactions on Information Systems, Vol. 27, No. 3, Article 18 pp. 1-31, May 2009

[9] T. Zhang, Automatic Singer Identification, Proceedings of the 2003 International Conference on Multimedia and Expo, May 2003

[10] A. Mesaros, T. Virtanen, A. Klapuri, Singer Identification in Polyphonic Music using Vocal Separation and Pattern Recognition Methods, International Conference on Music Information Retrieval, Vienna, Austria, 2007

[11] E. K. Youngmoo, B. Whitman, Singer Identification in Popular Music Recordings Using Voice Coding Features ,Proceedings of the 3rd International Conference on Music Information Retrieval. Paris, France: IRCAM, pp. 164-169, May 2002.

[12] R. C. Veltkamp, Content Based Image Retrieval System: A Survey,Technical Report, University of Utrecht, 2002. 